\title{
Real-time and high-performance calibration method for high-speed swept-source optical coherence tomography
}

\author{
Ehsan Azimi \\ Bin Liu \\ Mark E. Brezinski \\ Brigham and Women's Hospital \\ Department of Orthopedic Surgery \\ 75 Francis Street \\ Boston, Massachusetts 02115 \\ and \\ Harvard Medical School \\ 25 Shattuck Street \\ Boston, Massachusetts 02115
}

\begin{abstract}
For high-speed swept-source optical coherence tomography (SS-OCT), the real-time calibration process to convert the OCT signal to wave number space is highly essential. A novel calibration process/algorithm using a genetic algorithm and precise interpolation is developed. This algorithm is embedded and validated in a SS-OCT system with $16-\mathrm{kHz}$ A-scan rate. The performance of the new algorithm is evaluated by measuring point spread functions at two distinct locations in the entire imaging range. The data is compared to the same system but embedded with a regular calibration algorithm, which demonstrates about $20 \%$ improvement in the axial resolution. The steady improvement at different locations of the range suggests the strong robustness of the algorithm, which will ultimately optimize the operation performance of this SS-OCT system in terms of resolution and dynamic range and improves details in biological tissues. ( 2010 Society of Photo-Optical Instrumentation Engineers. [DOI: 10.1117/1.3285660]
\end{abstract}

Keywords: coherence; optical coherence tomography; algorithms; calibration; plaque rupture; arthritis.

Paper 09209RR received May 27, 2009; revised manuscript received Nov. 3, 2009; accepted for publication Nov. 9, 2009; published online Jan. 28, 2010.

\section{Introduction}

Optical coherence tomography (OCT) is a minimally invasive imaging technique that uses low-coherence gating to obtain up to submicron-level resolution, cross-sectional images of tissue microstructures. ${ }^{1-4}$ With early embodiments, the OCT technology emerged as a time-domain (TD) interferometry in terms of TD-OCT. Recently, spectral-domain OCT (SD-OCT) techniques have attracted a considerable amount of attention. $^{1,5-12}$ For the SD-OCT, which generally does not need mechanical movement in the reference arm of the interferometer, the spectral interferogram is measured, and the depth/amplitude information from the sample is obtained via the inverse Fourier transform of the interferogram in the wave number space. Fourier domain OCT (FD-OCT) and sweptsource OCT (SS-OCT) are two subtypes of the SD-OCT depending on the methods of spectral interferogram recording. (The nomenclature is consistent with the literature. However, the authors believe that the term "Fourier domain" is better than "spectral domain" to generalize the technique, since both the spectrometer and swept-source systems are based on Fourier transformation for image reconstruction. In a spectrometer-based system, spectral (wavelength) interference is detected directly with a dispersive element. Hence, it is suitably named "spectral domain." However, in a sweptsource system, the spectral interference is not measured directly, since the interference is recorded as a function of time instead of spectrum (and this is the motivation of this paper).

Address all correspondence to: Mark E. Brezinski, Department of Orthopedic Surgery, Brigham and Women's Hospital, 75 Francis Street, Boston, Massachusetts 02115. Tel: 617-233-2802; Fax:617-525-8356; Email: mebrezin@mit.edu.
It is imprecise to use "spectral domain" to generalize both systems. However, we have kept consistent with the literature to avoid confusion.) The former still uses a wideband light source similar to the one in the TD-OCT, while the spectral interferogram is recorded by a spectrometer at the exit of the interferometer. The latter uses a sweeping/tunable laser source, and the spectral interferogram is measured sequentially by time. Most recently, researchers has shown substantial interest in the SS-OCT because of its potential on ultrahigh scanning rate. ${ }^{8,10,12-16}$ We recently demonstrated that at least with current embodiments of the SD-OCT, performances of the SD-OCT in terms of dynamic range and penetration could be inferior to that of the TD-OCT. ${ }^{17,18}$ As one of our serial studies for characterizing the SD-OCT technique, the work presented here will focus on the issue of real-time spectral calibration for the SD-OCT, specifically for high-speed SS-OCT.

Typically, in SS-OCT, the instantaneous output of the light source is near-monochromatic and the wavelength is rapidly swept/tuned. This means that the actual obtained spectral interferogram is a function of time, but not a function of the wave number $k$. Before the inverse Fourier transform is performed for retrieving the depth-dependent A-scan profile, this function needs to be transformed into $k$ space, which is generally referred as the "calibration process." If the laser source can tune with constant speed in $k$ space, ${ }^{11}$ or the $k$ - $t$ curve is known a priori, and the spectrum sweeping is substantially stable and repeatable, the calibration might be needed only before operation. However, many laser sweeping mechanisms

1083-3668/2010/15(1)/016005/8/\$25.00 ○ 2010 SPIE 
involve mechanical movement or resonance, which inevitably raises concerns and real issues on the stability and repeatability of the spectrum. $8,10,12,13,15,16,19$ Thus, a real-time calibration is highly required, particularly in improving the imaging resolution in biological tissue. Poor calibration could ultimately cost the axial resolution and the ranging accuracy of the system, as demonstrated theoretically and experimentally in this work.

For calibration purposes, a variety of methods have been explored. These include:

1. Using a fixed filter to pick up a specific wavelength as a point reference. ${ }^{20-22}$ This method can dynamically compensate the instability of the starting point of the sweeping but requires high repeatability of the spectrum.

2. A Fabry-Pérot (FP) interferometer is employed to generate the frequency/wave number comb. ${ }^{14,23}$ While the laser wavelength is sweeping, the generated frequency/wave number is a series of pulses with fixed frequency interval between two adjacent pulses.

3. Similar to the FP method, a Mach-Zehnder interferometer (MZI) is used to generate the frequency clock. ${ }^{15,19}$ This is also the calibration reference for the system designated for this study. Different from the FP clock, the MZI clock is a sinusoid-type fringe. This means that the crossing points can also be referenced, which provides twice as many calibration points as an FP clock with the same free spectral range (FSR). In addition, the balanced detection technique can be used to reduce phase errors in the clock signal. In this work, we improve beyond the capabilities of these described approaches. Several practicalities are addressed with our approach. First, it might be challenging to perform the calibration in real time, since the calibration is usually done by the softwarespecifically, in the scenarios of high-speed or ultra-high-speed operation. A real-time search algorithm for cross-points, peak points, and trough points in the calibration signal is developed and optimized by the genetic algorithm (GA). Second, many sophisticated swept-laser sources provide a nonlinear $k$ - $t$ relationship, which suggests out the OCT signal is nonuniformly distributed in $k$ space. Poor or imprecise calibration could significantly degrade the system performance in terms of the resolution and the ranging accuracy. One study reported a hardware-based calibration by clocking the A/D converter with an uneven sampling in time to compensate for the nonlinear sweeping operation. ${ }^{19}$ This can reduce the time consumption of software calibration but increases the overall cost, as the electronics are much more complex, and is not feasible for different operation frequencies of the source. Our work develops a software-based real-time calibration process with advanced algorithms; the performance in terms of the full width half maximum (FWHM) width of point spread function (PSF) and the dynamic range is tested at different A-scan depth and compared with regular calibration algorithms as well. The presented algorithms are fast and robust, which minimizes the errors and thereby increases the resolution and dynamic range of the whole system.

\section{Methods}

\subsection{Theory}

The theoretical analysis of an SD-OCT/SS-OCT system is most easily based on a generic free-space Michelson interfer-

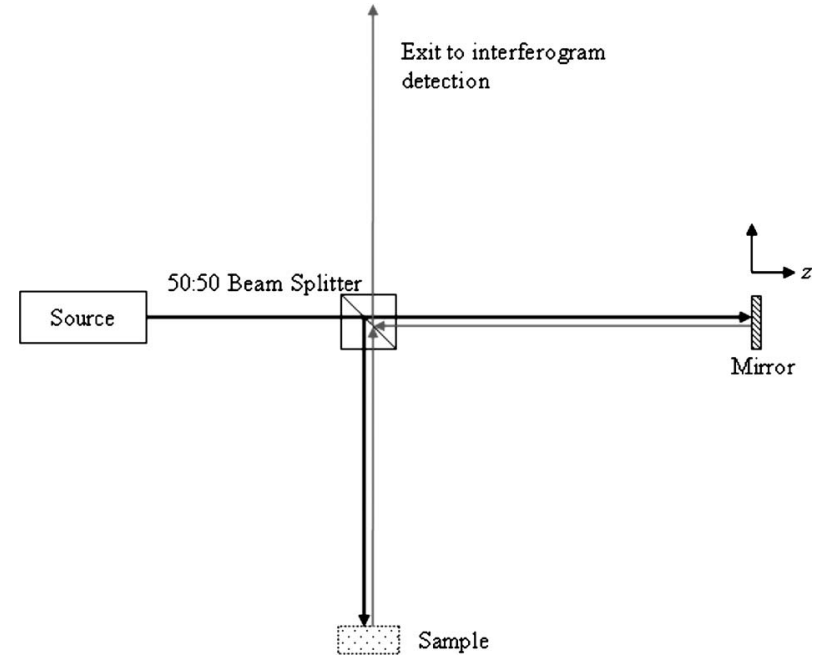

Fig. 1 Schematics of a free-space Michelson interferometer.

ometer, as depicted in Fig. 1, regardless of the differences in interferogram measurement or whether it is configured in free space or fiber-optically. In Fig. 1, the 50:50 beamsplitter evenly divides the light beam from the light source into the reference arm and the sample arm of the interferometer. In the reference arm, the light beam is returned by a mirror with amplitude reflectivity $r_{R}$. The sample beam is projected onto the sample, usually being focused to provide designated lateral resolution. Fresnel reflections occur at any discontinuities of refractive index within the sample. These reflected waves carrying information about the microstructures of sample are collected and returned to the beamsplitter. Both returned beams are combined at the beamsplitter and then generate an interferogram at the exit of the interferometer. To simplify this theoretical analysis, it is assumed that there are no insertion losses, dispersion, or polarization effects in these optical paths. The incident light field at the coupler is usually described as a complex function whose real part represents the real light disturbance and is conjugated with the imaginary part through Hilbert transform: ${ }^{1,24}$

$$
E_{0}(t)=\int_{0}^{\infty} a_{0}(\nu) \exp \left\{i\left[\phi_{0}(\nu)-2 \pi \nu t\right]\right\} \mathrm{d} \nu,
$$

where $a_{0}(\nu)$ and $\phi_{0}(\nu)$ represent the real-value amplitude and phase spectrum of the incident light, respectively, and $\nu$ is the frequency of the light. Thus, the intensity of this beam is represented by

$$
I_{0}=\frac{1}{2}\left\langle E_{0}(t) E_{0}^{*}(t)\right\rangle=\int_{-\infty}^{\infty} S(\nu) \mathrm{d} \nu=2 \int_{0}^{\infty} S(\nu) \mathrm{d} \nu,
$$

where $S(\nu)$ represents the power spectrum. According to the Weiner-Khinchin theorem, the autocorrelation function of the incident beam is the inverse Fourier transform of this spectrum as 


$$
\Gamma_{0}(\tau)=\int_{-\infty}^{\infty} S(\nu) \exp (i 2 \pi \nu \tau) \mathrm{d} \nu,
$$

which is a real-value function of time $\tau$, considering the symmetry between the positive-frequency part and the negativefrequency part of $S(\nu)$. It is important to notice that, in practice, the measured or generated light spectrum spans only the positive-frequency range in the form $2 S(\nu)$.

For the SD-OCT techniques, the mirror in the reference arm is in a static position. The spectral interferogram is measured either by placing a spectrometer at the exit or by sweeping out the spectrum $S(k)$ as a function of wave number $k$. The former approach is usually referred to as the Fourier domain OCT (FD-OCT), and the latter as the swept-source OCT (SS-OCT). Accordingly, the individual spectral components of the light field from both the reference arm and the sample arm can be respectively described as

$$
E_{R}(t, k)=\frac{1}{2} r_{R} a_{0}(k) \exp \left[j \phi_{0}(k)\right] \exp (j 2 \pi \nu t) \exp \left(-j 2 \pi k z_{R}\right)
$$

$$
\begin{aligned}
E_{S}(t, k)= & \frac{1}{2} a_{0}(k) \exp \left[j \phi_{0}(k)\right] \exp (j 2 \pi \nu t) \\
& \times \int_{-\infty}^{\infty} r_{S}(z) \exp (-j 2 \pi k z) \mathrm{d} z
\end{aligned}
$$

The phase term in the integral of Eq. (4) is introduced by the reference mirror at $z_{R}$ away from the coupler. The coefficient $r_{R} / 2$ reflects the fact that the amplitude reflectivity of this mirror is set as $r_{R}$, and the light beam is split twice by the splitter. Similarly, in Eq. (5), $r_{S}(z)$ represents the scattering potential of the sample and is assumed to be independent on the wavelength. $z$ is measured from the coupler. The integral over $z$ reflects all the contributions to the returned sample beam from the scattering potential. The intensity of each spectral component at the exit of the interferometer is

$$
\begin{aligned}
I_{D}(k)= & \frac{1}{2}\left\langle\left[E_{R}(t, k)+E_{S}(t, k)\right]\left[E_{R}(t, k)+E_{S}(t, k)\right]^{*}\right\rangle \\
= & \frac{1}{2}\left\langle E_{R}(t, k) E_{R}^{*}(t, k)\right\rangle+\frac{1}{2}\left\langle E_{S}(t, k) E_{S}^{*}(t, k)\right\rangle \\
& +\frac{1}{2}\left\langle E_{R}(t, k) E_{S}(t, k)^{*}\right\rangle+\frac{1}{2}\left\langle E_{S}(t, k) E_{R}(t, k)^{*}\right\rangle .
\end{aligned}
$$

Accordingly,

$$
\begin{gathered}
I_{R}(k)=\frac{1}{2}\left\langle E_{R}(t, k) E_{R}^{*}(t, k)\right\rangle=\frac{1}{4} S(k) r_{R}^{2}, \\
I_{S}(k)=\frac{1}{2}\left\langle E_{S}(t, k) E_{S}^{*}(t, k)\right\rangle=\frac{1}{4} S(k)\left|F T\left[r_{S}(z)\right]\right|^{2},
\end{gathered}
$$

$$
\begin{aligned}
& \frac{1}{2}\left\langle E_{R}(t, k) E_{S}(t, k)^{*}\right\rangle+\frac{1}{2}\left\langle E_{S}(t, k) E_{R}(t, k)^{*}\right\rangle \\
& =\frac{1}{4} S(k) r_{R}\left\{F T\left[r_{S}\left(z-z_{R}\right)\right]+F T\left[r_{S}\left(z_{R}-z\right)\right]\right\} .
\end{aligned}
$$

The abbreviation FT in Eq. (7) stands for Fourier transform operation. Substituting Eq. (7) into Eq. (6), the spectral interferogram at the exit can then be described as

$$
\begin{aligned}
I_{D}(k)= & \frac{1}{4} S(k)\left\{r_{R}^{2}+r_{R} F T\left[r_{S}\left(z-z_{R}\right)\right]+r_{R} F T\left[r_{S}\left(z_{R}-z\right)\right]\right\} \\
& \left.+\left|F T\left[r_{S}(z)\right]\right|^{2}\right\} .
\end{aligned}
$$

By taking the inverse Fourier transform on this spectral interferogram, a real-value function of single variable $z$ can be obtained:

$$
\begin{aligned}
F T^{-1}\left[I_{D}(k)\right]= & \frac{1}{4} F T^{-1}[S(k)] \otimes\left(r_{R}^{2} \delta(z)+r_{R} r_{S}\left(z-z_{R}\right)\right. \\
& \left.+r_{R} r_{S}\left(z_{R}-z\right)+F T^{-1}\left\{\left|F T\left[r_{S}(z)\right]\right|^{2}\right\}\right) .
\end{aligned}
$$

In Eq. (9), the inverse Fourier transform of the spectrum is the autocorrelation function $\Gamma_{0}(z)$ in $z$ space, which is convoluted with the four terms in brackets. The first term in brackets, a delta function, along with the last term that is equivalent to the autocorrelation of the scattering potential $r_{S}(z)$, exists around $z=0$. The second and the third term in brackets are replicas of the scattering potential, but all shifted to $z=0$ by $z_{R}$, and symmetrical to $z=0$. Since the designated scattering potential is putatively prominent around $z_{R}$, the scattering potential might therefore be retrieved around $z=0$. In addition, if the mirror position in the reference arm, $z_{R}$, is set outside the regime of the sample, the second and the third term in brackets can be easily distinguished from each other. Thus, either term can represent the designated scattering potential. It is useful to notice that, practically, the sweeping or measurement of the spectrum obtains only the positive wave number part of the spectral interferogram in Eq. (8). This means that the inverse Fourier transform as Eq. (9) will generate a complex function. Therefore, the modulus has to be calculated for retrieving $r_{S}(z)$

Practically, in SS-OCT, the spectrum is swept in the time domain. This means that the actual obtained spectral interferogram of the detector is intended to perform linearly in the time domain. So, $I_{D}(t)$ is a function of time $I_{D}(t)$ instead of the wave number $I_{D}(k)$. This function needs to be transformed into $k$ space before the inverse Fourier transform is performed Eq. (9). Clearly then, the relationships between the wave number $k$ and the sweeping time $t$ in terms of a function $k$ $=f(t)$ must be taken into account. Then, the interferogram in $k$ space can be obtained as $I_{D}\left[f^{-1}(k)\right]$. This process is generally referred to as the calibration in SD-OCT. If a strict linear relationship could be achieved between $k$ and $t$ as

$$
k=\frac{\Delta k}{\Delta T} t+k_{0}
$$

where $\Delta T$ is the sampling window in the time domain, $\Delta k$ is the wave number range in the time interval $\Delta T, \Delta k / \Delta T$ rep- 
resents the constant sweeping speed, $k_{0}$ is the center wave number, and $t$ starts from $-\Delta T / 2$ to $\Delta T / 2$, then the $k$-space interferogram presents as a function of time $t$ :

$$
\begin{aligned}
I_{D}(t)= & \frac{1}{4} S\left(\frac{\Delta k}{\Delta T} t-k_{0}\right)\left\{r_{R}^{2}+r_{R} F T\left[r_{S}\left(z-z_{R}\right)\right]\right. \\
& \left.+r_{R} F T\left[r_{S}\left(z_{R}-z\right)\right]+\left|F T\left[r_{S}(z)\right]\right|^{2}\right\} .
\end{aligned}
$$

The inverse Fourier transform on this time-sequential interferogram is

$$
\begin{aligned}
F T_{t}^{-1}\left[I_{D}(t)\right] \propto & \Gamma_{0}\left(\frac{\Delta T}{\Delta k} f\right) \otimes\left\{r_{R}^{2} \delta\left(\frac{\Delta T}{\Delta k} f\right)+r_{R} r_{S}\left(\frac{\Delta T}{\Delta k} f-z_{R}\right)\right. \\
& +r_{R} r_{S}\left(z_{R}-\frac{\Delta T}{\Delta k} f\right) \\
& \left.+F T_{t}^{-1}\left\{\left|F T_{t}\left[r_{S}\left(\frac{\Delta T}{\Delta k} f\right)\right]\right|^{2}\right\}\right\} .
\end{aligned}
$$

In Eq. (12), the subscript $t$ in abbreviations of Fourier transform marks that the transform is between time domain and frequency $f$ domain reciprocally. Equation (12) indicates that, for such a linear relationship, the inverse Fourier transform can be directly applied on $I_{D}(t)$. Consequently, the designated profile can then be retrieved with just rescaling the data in the $z$ dimension. However, many kinds of sophisticated sweptlaser sources usually provide a near-linear relationship between the wavelength $\lambda$ and time $t$ such as

$$
\lambda=\frac{\Delta \lambda}{\Delta T} t+\lambda_{0}
$$

Similarly, $\Delta \lambda / \Delta T$ represents the constant wavelength sweeping speed, $\lambda_{0}$ is the central wavelength, $t$ starts from $-\Delta T / 2$ to $\Delta T / 2$. This linearity in $\lambda$ reflects the nonlinearity between $k$ and $t$ because

$$
t=\frac{\Delta T}{\Delta \lambda}\left(\lambda-\lambda_{0}\right)=\frac{\Delta T}{\Delta \lambda}\left(\frac{1}{k}-\frac{1}{k_{0}}\right) .
$$

Expanding the part in parentheses into its power series around $k_{0}$, Eq. (14) can become

$$
t=\frac{\Delta T}{\Delta \lambda}\left[-\frac{1}{k_{0}}\left(\frac{k}{k_{0}}-1\right)+\frac{1}{k_{0}}\left(\frac{k}{k_{0}}-1\right)^{2}-\frac{1}{k_{0}}\left(\frac{k}{k_{0}}-1\right)^{3}+\cdots\right] .
$$

Equation (15) indicates that this nonlinearity, which is frequently referred to as an issue of nonuniform sampling in the $k$ space literature, ${ }^{25,26}$ will introduce a series of nonlinear terms in the converted spectral interferogram in $k$-space, which will inevitably cause distortions in the autocorrelation $\Gamma_{0}(z)$, and significantly cost the spatial resolution and ranging accuracy as will be experimentally demonstrated in later sections. If a priori knowledge exists, the calibration may need to be performed only before each OCT operation. Unfortunately, this is not the case for many laser sweeping mechanisms, particularly with respect to the stability and repeatability of the spectrum sweeping ${ }^{8,10,12,13,15,16,19}$-for example, a common mechanism of irregularities in the SS-OCT is using the

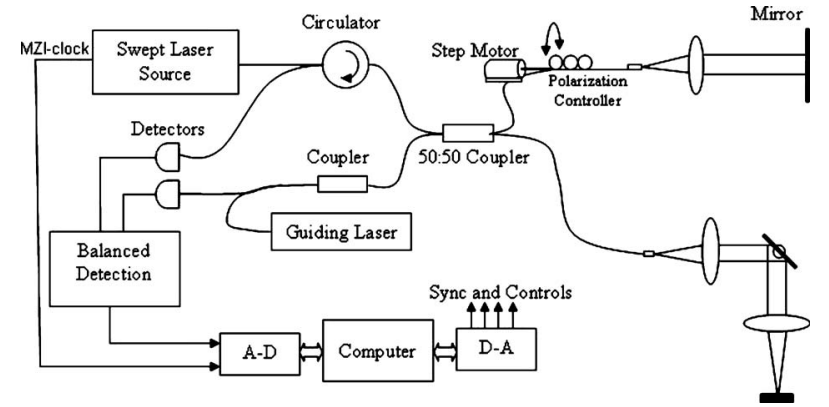

Fig. 2 System configuration diagram of the designated SS-OCT.

piezoelectric transducer (PZT) resonator in the tuning mechanism. The hysteresis of the PZT affects repeatability of the spectrum. In addition, the stability errors can be caused by a variety of sources, such as the jitter noise in PZT driving signals. Thus, a real-time calibration is highly desirable and demanded.

In this designated SS-OCT system, a Mach-Zehnder interferometer (MZI) is used for the real-time calibration/ compensation purposes. An MZI is a particularly simple twobeam interferometer. The intensity of its output periodically changes if the wavelength of the monochromatic or quasimonochromatic light source at the input is scanning. This signal shows maxima and minima that are equally spaced in the optical frequency domain or equivalent wave number domain ( $k$-space). The difference between two maxima is defined by the free spectral range (FSR) of the interferometer, which is determined by the optical path length mismatch between both arms in the MZI. In this work, this is set at $\sim 100 \mathrm{GHz}$, which corresponds to a wave number interval of $\sim 3.33(\mathrm{~cm})^{-1}$. In practice, zero-crossings in the electronic output signal of MZI are used, as demonstrated in this work. In an SS-OCT system, the wavelength of the laser source is rapidly tuned. As these points are determined in time sequence, they are used to index the data set of the simultaneously captured spectral interferogram. Thus, the OCT signal is transformed into a set of data with a fixed wave number interval before the fast Fourier transform.

\subsection{Experiment}

The general optical arrangement of the high-speed SS-OCT designated for this study is depicted in Fig. 2, where a fiberoptic Michelson interferometer plays the core architecture. The swept-laser source (Thorlabs SL1325-P16,Newton, New Jersey) provides $\sim 100$-nm (FWHM) wavelength scan range around $1325-\mathrm{nm}$ central wavelength, as well as $10-\mathrm{mW}$ output power. The wavelength sweeping rate is $16 \mathrm{kHz}$, and the instantaneous coherence length of the laser is measured to be $>7 \mathrm{~mm}$. In this laser source, a Mach-Zehnder interferometer is embedded to provide a frequency clock with $100-\mathrm{GHz}$ optical frequency space $(\sim 0.6 \mathrm{~nm}$ in wavelength). It is output as an analog clock signal for real-time spectrum calibration in the SS-OCT. The light beam from the source is guided into a circulator and a $2 \times 2$ 50:50 fiber coupler. The two output ports of the coupler compose the two arms of the Michelson interferometer. One arm, the reference arm, is projected by a fiber collimator into a mirror, which is movable along the 


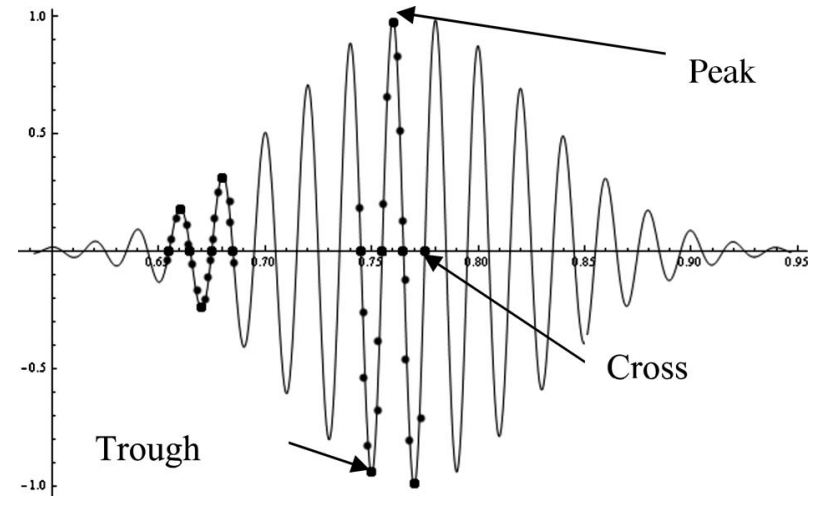

Fig. 3 Illustrative frequency clock signal from the Mach-Zehnder interferometer. Gray points are the outcome of A/D sampling. Black points represent to-be-found cross, peak, and trough points.

beam direction through a microstage (Thorlabs, Newton, New Jersey). In the reference arm, a motorized Lefevre-type polarization controller is built and implemented for polarization measurement with this SS-OCT. The sample arm is scanned by an X-Y scanner (Cambridge Technology, Cambridge, Massachusetts). The scanned beam is focused on the sample through an objective lens with $30-\mathrm{mm}$ focal length. This X-Y scanner is synchronized with the laser sweeping to provide the B-scan and 3-D scan for the OCT imaging. The returned light beams combine and interfere at the coupler. The interferograms from the two ports of the coupler are guided into a dual-balanced detector (Thorlabs PDB-145C, Newton, New Jersey), the circulator and another coupler, respectively. This coupler has 95:5 splitting ratio and also helps to guide the aiming laser beam $(632 \mathrm{~nm})$ to both arms.

The signal processing unit consists of a computer (T7400, Dell, Round Rock, Texas) with dual-core $2.66-\mathrm{GHz}$ processor, a dual-channel A/D card with 180-MS/s acquisition rate and 16-bit resolution on PCI Express Bus (ATS-9462, Alazer Tech, Pointe-Claire, Quebec, Canada.) This card is operated in a mode with parallel and synchronous acquisition of two channels for recording the interference signal from the dual balanced detector and frequency clock signal from the MachZehnder interferometer. The control signal for the X-Y scanner and the system synchronization are generated through a D/A card with 1-MS/s sampling rate and 16-bit resolution (NI-6731, National Instrument, Austin, Texas).

The software was developed in the object-oriented paradigm using Microsoft Visual $\mathrm{C}++$ to perform data processing, recalibration, imaging reconstruction, and hardware control. Using the state-of-the-art overlapped I/O structure, the data is processed without prior buffering. It is beneficial comparing with the traditional synchronous function execution, where it does not return until the operation has been completed. This means that the execution of the calling thread can be blocked for an indefinite period while it waits for a time-

(a)

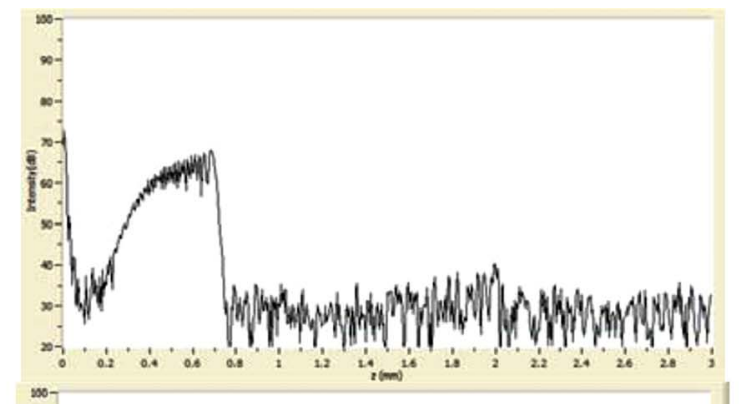

(b)
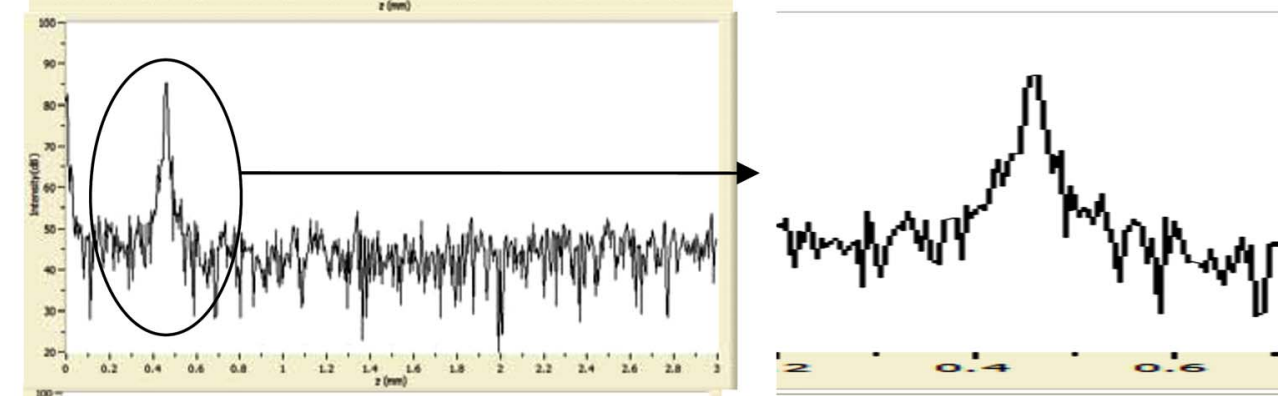

(c)

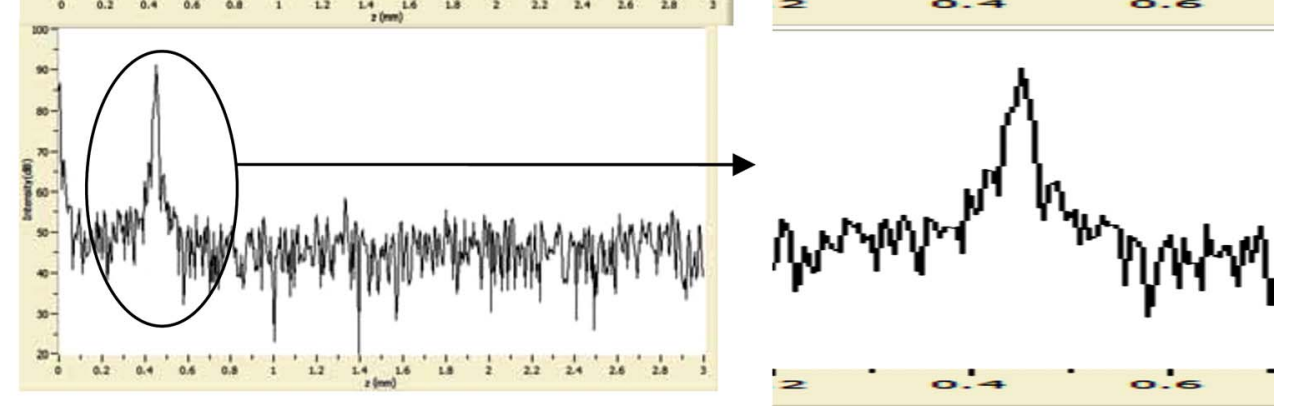

Fig. 4 Sample A-scans of a mirror at 0.45-mm distance: (a) without calibration, (b) with regular calibration, and (c) with the calibration developed in this work [(b) and (c) are magnified for better comparison]. 
(a)

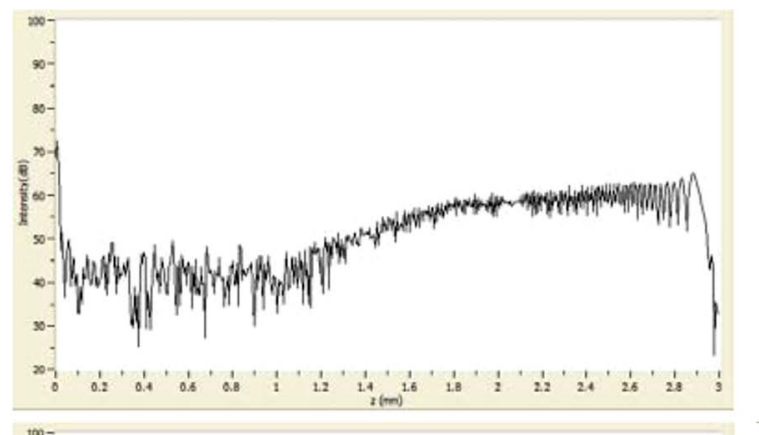

(b)

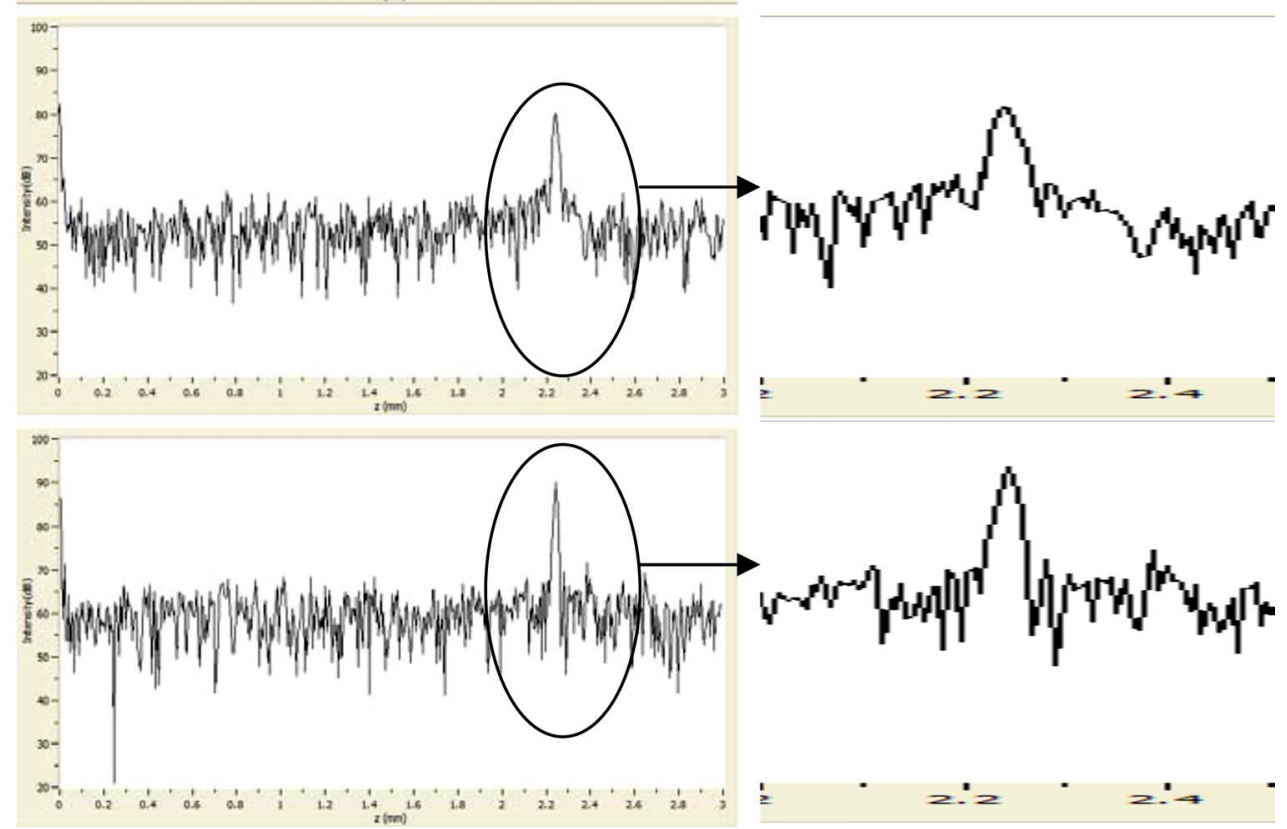

Fig. 5 Sample A-scans of a mirror at 2.2-mm distance: (a) without calibration, (b) with regular calibration, and (c) with the calibration developed in this work [(b) and (c) are magnified for better comparison].

consuming operation to finish. Functions called for overlapped operation can return immediately, even though the operation has not been completed. This enables a timeconsuming I/O operation to be executed in the background while the calling thread is free to perform other tasks. For example, a single thread can perform simultaneous I/O operations on different handles, or even simultaneous read and write operations on the same handle. This, along with advanced multithreading, improves the speed and real-time image generation.

An illustrative frequency clock signal from the MZI is shown in Fig. 3. This interferometric signal is evenly sampled by the A/D card, simultaneously with the interference signal from the Michelson interferometer. As described earlier, the clock signal is used as a reference, with its cycles being equidistant in frequency. The difference between two maxima is defined by the FSR of the MZI.

In one previous study, recalibration was performed using a fast nearest-neighbor check algorithm, which is referred to as the regular calibration here. ${ }^{14} \mathrm{~A}$ fast search algorithm for peak points and trough points in the calibration signal was performed. If the samples satisfied the conditions of the algorithm, the corresponding points in the OCT signal were added to the recalibrated signal array. While this method provides speed that is adequate for real-time preview, it fails to determine the actual peaks and troughs and leads to errors. In our study, an algorithm is developed that not only minimizes the errors caused by the aforementioned event, but also generates the image in real-time. Additionally, it doubles the number of samples used for the inverse Fourier transform, and thereby increases the fidelity in reconstruction of the signal and enables larger imaging depth.

We used a genetic algorithm (GA) to optimize the search method in the calibration trace. An appropriate fitness function is defined in a way that local extremes and vicinity to zero increase the fitness value for extremes (peaks and troughs) and cross-points, respectively. The value of the Gaussian envelope of the calibration signal in proximity of each extreme is used as an auxiliary tool for the fitness function. Using this data set as the first generation, during each successive generation, a number of points are selected based on their quality measured by fitness function. Consecutive iterations of this method would increase the average fitness of the population of the next generation. (Multiple generations are achieved in microseconds.) This generational process would continue until the requisite number of points with desired quality is attained. At this point, the algorithm is terminated. These points are, afterward, used for interpolation. 
In order to find the actual extremes and cross-points, two interpolations are performed on the obtained data. Cubic spline interpolation is performed for the extremes and interference signal, while linear interpolation is done for the crosspoints. The functions used here for extremes and interference signal must behave smoothly to avoid the jitter noise. To satisfy this condition, the functions must be differentiable, and their second derivative must be continuous. ${ }^{27}$ Considering this fact and also to minimize the interpolation error and avoid Runge's phenomenon, spline functions are used here that normally satisfy these requirements. It should be noted that the samples are acquired in a rate that guarantees that there is only one extreme in each interpolation.

Real-time display requires a fast recalibration algorithm. Using a multithreading technique, we can perform inverse Fourier transform, GA, and interpolation in parallel for the axial scans, and therefore they do not increase the overall time of the process. This method increases the performance of the system in different ways. Adding cross-points to the reference points leads to doubled sampling frequency and higher accuracy in signal reconstruction; it will minimize the errors in finding the reference points by the GA-based optimized search and precise interpolation. As illustrated in the experimental results, resolution, dynamic range, and image quality are improved by using the proposed method in an SS-OCT system.

\section{Results and Discussion}

To test its efficacy and robustness, this real-time calibration process/algorithm is implemented and embedded in the described SS-OCT system. A-scans of a mirror placed in the sample arm, in terms of point spread function (PSF) width and total intensity, are measured with $16-\mathrm{kHz}$ rate. The designed imaging range of this system is 0 to $3 \mathrm{~mm}$. To investigate the efficacy and robustness of calibration along the whole imaging range, the PSF is measured at two distant locations: proximal (around 0.45 -mm z-offset) and distal (around 2.2-mm $z$-offset), respectively. At each position, six measurements are conducted. The same testing procedures are performed on the same OCT system, but with the regular calibration algorithm. The FWHM of the PSF (resolution) and the dynamic range is measured from those A-scans. The two groups of data (calibrated with the presented algorithm, and calibrated with the regular one) are compared and analyzed.

Figure 4 gives sample A-scans at $0.45-\mathrm{mm}$ distance: (a) without calibration, (b) with conventional calibration, and (c) with calibration developed in this work. In Fig. 4(a) a largely spread PSF can be seen, which is due to the nonlinear $k-t$ relations of spectrum sweeping, or equivalently the nonuniform distributions of sampled signals in $k$-space. As pointed out in Sec. 2.1, all the nonlinear terms in the power series representing the spectrum in $k$-space will contribute to this broadening. This is also supported by the observed asymmetry of the PSF. Figure 4(b) shows a PSF of an SS-OCT with the currently used calibration algorithm ${ }^{14}$ whose FWHM width is about $14 \mu \mathrm{m}$ and dynamic range is around $38 \mathrm{~dB}$. Compared with Fig. 4(b), the PSF in Fig. 4(c) demonstrates reduced FWHM and increased range, which optimizes the system performance in terms of axial resolution and dynamic range. The analysis of the data sets from the repeated measurements (six
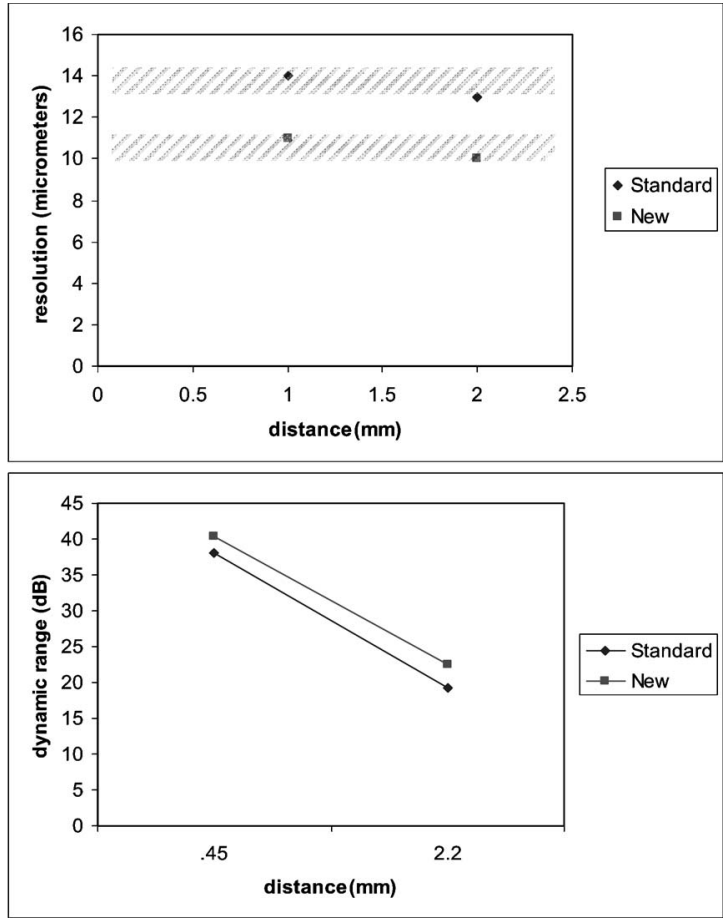

Fig. 6 Comparison of the performance of the standard (lozenge) and new (square) algorithm: (a) resolution (the dashed area represents measurement accuracy); (b) dynamic range.

rounds) show distinct improvements by this work. The average FWHM of PSF and the dynamic range with regular calibration is $14 \mu \mathrm{m}$ and $38 \mathrm{~dB}$, respectively. The ones with presented calibration are $11 \mu \mathrm{m}$ and $40.4 \mathrm{~dB}$, respectively.

Similar outcomes can be seen from Fig. 5, which are A-scans captured at $2.2 \mathrm{~mm}$ : (a) without calibration, (b) with regular calibration, and (c) with calibration developed in this work. Specifically, the much wider PSF in Fig. 5(a) reflects the fact that the nonlinearity issue will be much more serious at the far side of the imaging range. The decreases of the dynamic range in Figs. 5(b) and 5(c) are largely due to the modulation of the shape of the coherent wave pack of the light source, which is determined by the instantaneous line width of the emitted laser. ${ }^{17}$ The FWHM of PSF and the dynamic range with the regular calibration is $13 \mu \mathrm{m}$ and $19.2 \mathrm{~dB}$, respectively. The ones with new calibration process are $10 \mu \mathrm{m}$ and $22.5 \mathrm{~dB}$, respectively.

Figure 6 illustrates how the resolution and dynamic range change with depth in both the standard and new approach (lozenge and square, respectively). Better resolution and higher dynamic range is achieved via GA and spline interpolation. There is a measurement error associated with digitization (one pixel error), which is shown in the resolution graph as a dashed area.

\section{Conclusion}

The theoretical analysis in this work demonstrates that any nonlinearity between swept frequency/wave number and sweeping time in an SS-OCT could significantly deteriorate the axial resolution and the ranging accuracy of the system. More effective methods for compensating nonlinearities due 
to vector space conversion and source sweep rates are needed with SS-OCT. A real-time calibration process/algorithm using a genetic algorithm and precise interpolation is developed by the work presented here. This algorithm is embedded and validated in an SS-OCT system with $16-\mathrm{kHz}$ A-scan rate and 8 - $\mu \mathrm{m}$ theoretical resolution. The performance of the new algorithm is tested by measuring A-scans of a mirror. Compared to the same system but embedded with a regular calibration algorithm, the presented method can improve the axial resolution about $20 \%$. There is also a 5 to $10 \%$ improvement in dynamic range. Improvements are noted in both superficial and deep imaging. The testing at the close and far end of the imaging range demonstrates that the improvement is steady along the whole range, which suggests the strong robustness of the algorithm. This work demonstrates a superior performance of this compensation technique over the most effective one currently available and is likely to translate into improved imaging of microstructures.

\section{Acknowledgments}

Dr. Brezinski's work is currently funded by the National Institutes of Health Grant Nos. R01 AR44812, R01 HL55686, R01 EB02638/HL63953, R01 AR46996, and R01 EB000419.

\section{References}

1. M. E. Brezinski, Optical Coherence Tomography: Principle and Practice, Academic Press, Burlington, MA (2006).

2. M. E. Brezinski and J. G. Fujimoto, "Optical coherence tomography: high-resolution imaging in nontransparent tissue," IEEE J. Sel. Top. Quantum Electron. 5(4), 1185-1192 (1999).

3. G. J. Tearney, M. E. Brezinski, B. E. Bouma, S. A. Boppart, C. Pitris, J. F. Southern, and J. G. Fujimoto, "In vivo endoscopic optical biopsy with optical coherence tomography," Science 276(5321), 2037-2039 (1997).

4. M. E. Brezinski, G. J. Tearney, B. E. Bouma, J. A. Izatt, M. R. Hee, E. A. Swanson, J. F. Southern, and J. G. Fujimoto, "Optical coherence tomography for optical biopsy - properties and demonstration of vascular pathology," Circulation 93(6), 1206-1213 (1996).

5. A. F. Fercher, C. K. Hitzenberger, G. Kamp, and S. Y. Elzaiat, "Measurement of intraocular distances by backscattering spectral interferometry," Opt. Commun. 117(1-2), 43-48 (1995).

6. S. R. Chinn, E. A. Swanson, and J. G. Fujimoto, "Optical coherence tomography using a frequency-tunable optical source," Opt. Lett. 22(5), 340-342 (1997).

7. H. Gerd and L. Michael Walter, "'Coherence radar' and 'spectral radar'-new tools for dermatological diagnosis," J. Biomed. Opt. 3(1), 21-31 (1998)

8. W. Y. Oh, S. H. Yun, G. J. Tearney, and B. E. Bouma, " $115 \mathrm{kHz}$ tuning repetition rate ultrahigh-speed wavelength-swept semiconductor laser," Opt. Lett. 30(23), 3159-3161 (2005).

9. E. Gotzinger, M. Pircher, R. A. Leitgeb, and C. K. Hitzenberger, "High speed full range complex spectral domain optical coherence tomography," Opt. Express 13(2), 583-594 (2005).

10. R. Huber, D. C. Adler, and J. G. Fujimoto, "Buffered Fourier domain mode locking: unidirectional swept laser sources for optical coher- ence tomography imaging at 370,000 lines/s," Opt. Lett. 31(20) 2975-2977 (2006).

11. C. M. Eigenwillig, B. R. Biedermann, G. Palte, and R. Huber, "Kspace linear Fourier domain mode locked laser and applications for optical coherence tomography," Opt. Express 16(12), 8916-8937 (2008).

12. M. Y. Jeon, J. Zhang, Q. Wang, and Z. Chen, "High-speed and wide bandwidth Fourier domain mode-locked wavelength swept laser with multiple SOAs," Opt. Express 16(4), 2547-2554 (2008).

13. R. Huber, M. Wojtkowski, and J. G. Fujimoto, "Fourier domain mode locking (FDML): a new laser operating regime and applications for optical coherence tomography," Opt. Express 14(8), 3225-3237 (2006).

14. R. Huber, M. Wojtkowski, K. Taira, J. G. Fujimoto, and K. Hsu, "Amplified, frequency swept lasers for frequency domain reflectometry and OCT imaging: design and scaling principles," Opt. Express 13(9), 3513-3528 (2005).

15. V. J. Srinivasan, R. Huber, I. Gorczynska, J. G. Fujimoto, J. Y. Jiang, P. Reisen, and A. E. Cable, "High-speed, high-resolution optical coherence tomography retinal imaging with a frequency-swept laser at $850 \mathrm{~nm}$, , Opt. Lett. 32(4), 361-363 (2007).

16. S. H. Yun, C. Boudoux, G. J. Tearney, and B. E. Bouma, "High-speed wavelength-swept semiconductor laser with a polygon-scanner-based wavelength filter," Opt. Lett. 28(20), 1981-1983 (2003).

17. B. Liu and M. E. Brezinski, "Theoretical and practical considerations on detection performance of time domain, Fourier domain, and swept-source optical coherence tomography," J. Biomed. Opt. 12(4), 044007 (2007).

18. K. Zheng, B. Liu, C. Y. Huang, and M. E. Brezinski, "Experimental confirmation of potential swept-source optical coherence tomography performance limitations," Appl. Opt. 47(33), 6151-6158 (2008).

19. R. Huber, M. Wojtkowski, J. G. Fujimoto, J. Y. Jiang, and A. E. Cable, "Three-dimensional and C-mode OCT imaging with a compact, frequency swept laser source at 1300 nm," Opt. Express 13(26), 10523-10538 (2005).

20. B. J. Vakoc, S. H. Yun, J. F. de Boer, G. J. Tearney, and B. E. Bouma, "Phase-resolved optical frequency domain imaging," Opt. Express 13(14), 5483-5493 (2005).

21. S. H. Yun, G. J. Tearney, B. E. Bouma, B. H. Park, and J. F. de Boer, "High-speed spectral-domain optical coherence tomography at $1.3 \mu \mathrm{m}$ wavelength," Opt. Express 11(26), 3598-3604 (2003).

22. J. Zhang and Z. P. Chen, "In vivo blood flow imaging by a swept laser source based Fourier domain optical Doppler tomography," Opt. Express 13(19), 7449-7457 (2005).

23. J. Zhang, J. S. Nelson, and Z. P. Chen, "Removal of a mirror image and enhancement of the signal-to-noise ratio in Fourier-domain optical coherence tomography using an electro-optic phase modulator," Opt. Lett. 30(2), 147-149 (2005).

24. M. Born and E. Wolf, Principles of Optics, Cambridge Univeristy Press, Cambridge, UK (2002).

25. M. Y. Jeon, J. Zhang, and Z. P. Chen, "Characterization of Fourier domain mode-locked wavelength swept laser for optical coherence tomography imaging," Opt. Express 16(6), 3727-3737 (2008).

26. M. Mujat, B. H. Park, B. Cense, T. C. Chen, and J. F. de Boer, "Autocalibration of spectral-domain optical coherence tomography spectrometers for in vivo quantitative retinal nerve fiber layer birefringence determination," J. Biomed. Opt. 12(4), 041205 (2007).

27. E. Azimi, M. Ghobadi, E. T. Esfahani, M. Keshmiri, and A. F. Tehrani, "Three-dimensional smooth trajectory planning using realistic simulation," Robocup 2004: Robot Soccer World Cup VIII 3276, 381-393 (2005). 